\title{
Les bibliothèques de La Pocatière et les programmes d'immersion
}

\section{The Libraries of La Pocatière and the Immersion Programmes Las bibliotecas de La Pocatière y los programas de inmersión}

\section{Patricia S. Kuntz}

Volume 53, numéro 3, juillet-septembre 2007

URI : https://id.erudit.org/iderudit/1030648ar

DOI : https://doi.org/10.7202/1030648ar

Aller au sommaire du numéro

Éditeur(s)

Association pour l'avancement des sciences et des techniques de la documentation (ASTED)

ISSN

0315-2340 (imprimé)

2291-8949 (numérique)

Découvrir la revue

Citer cet article

Kuntz, P. S. (2007). Les bibliothèques de La Pocatière et les programmes d'immersion. Documentation et bibliothèques, 53(3), 167-173.

https://doi.org/10.7202/1030648ar

\section{Résumé de l'article}

L'auteure présente un tour d'horizon des différentes bibliothèques que l'on trouve à La Pocatière : relations entre elles, personnels, crédits, automatisation, prêts, etc. Dans cette ville, on compte cinq bibliothèques qui restent ouvertes pendant la période des cours d'été : celles du Collège de Sainte-Anne, du Cégep de La Pocatière et de l'Institut de technologie agroalimentaire, la Bibliothèque municipale ainsi que les Archives de la Côte-du-Sud. La ville accueille les étudiants venus suivre un programme d'immersion en français durant l'été. L'auteure croit que ces bibliothèques pourraient offrir davantage de ressources documentaires et des services supplémentaires au cours de la période où est dispensé ce programme.
Tous droits réservés (c) Association pour l'avancement des sciences et des techniques de la documentation (ASTED), 2007
Ce document est protégé par la loi sur le droit d'auteur. L'utilisation des services d'Érudit (y compris la reproduction) est assujettie à sa politique d'utilisation que vous pouvez consulter en ligne. 


\title{
Les bibliothèques de La Pocatière et les programmes d'immersion
}

\author{
PATRICIA S. KUNTZ \\ University of Wisconsin, Madison \\ pskuntz@uwalumni.com
}

\section{RÉSUMÉ | ABSTRACTS | RESUMEN}

L'auteure présente un tour d'horizon des différentes bibliothèques que l'on trouve à La Pocatière: relations entre elles, personnels, crédits, automatisation, prêts, etc. Dans cette ville, on compte cinq bibliothèques qui restent ouvertes pendant la période des cours d'été: celles du Collège de Sainte-Anne, du Cégep de La Pocatière et de l'Institut de technologie agroalimentaire, la Bibliothèque municipale ainsi que les Archives de la Côte-du-Sud. La ville accueille les étudiants venus suivre un programme d'immersion en français durant l'été. L'auteure croit que ces bibliothèques pourraient offrir davantage de ressources documentaires et des services supplémentaires au cours de la période où est dispensé ce programme.

\section{The Libraries of La Pocatière and the Immersion Programmes}

The author describes the libraries of La Pocatiere, their personnel, budgets, automated systems, loans and relationships one to the other. There are five libraries open during the summer session : the library of the College de Sainte-Anne, the library of the CEGEP, the library of the Institut agro-alimentaire, the public library and the archives of the Côte-du-Sud. During the summer, the city hosts the students of a French immersion programme. The author believes that the libraries could offer additional resources and services when the programme is in session.

\section{Las bibliotecas de La Pocatière y los programas de inmersión}

El autor presenta una visión general de las bibliotecas de La Pocatière : personal, créditos, automatización, préstamos, relaciones interbibliotecarias, etc. Cinco de estas bibliotecas se encuentran abiertas durante el verano : la biblioteca del Colegio de Santa Ana, la del Cégep, la del instituto agroalimentario, la de la biblioteca municipal y la de los archivos de la costa sur. La ciudad alberga a estudiantes de un programa de inmersión en francés durante el verano. El autor considera que estas bibliotecas podrían ofrecer recursos y servicios adicionales junto con la implementación del programa.
T Raditionnellement, La Pocatière occupe une place très importante en tant que centre d'enseignement. Le Collège de Sainte-Anne (1827) et l'École d'agriculture (1859) - aujourd'hui l'Institut de technologie agroalimentaire - y ont été fondés au XIX ${ }^{\mathrm{e}}$ siècle, le premier par Charles-François Painchaud (1782-1838), la seconde par François Pilote (1811-1886). Depuis, ces écoles n'ont cessé d'attirer les étudiants d'un peu partout au Québec et d'ailleurs. En ce qui concerne les programmes d'immersion en français, signalons que déjà dans les années 1960, le Collège de Sainte-Anne a été le siège d'un programme destiné au Corps de la Paix (États-Unis) : les coopérants américains y venaient pour apprendre le français avant d'aller travailler dans divers pays en développement francophones.

Le Canada a mis sur pied, il y a environ 30 ans, des programmes d'immersion grâce auxquels des bourses ont été distribuées à ceux qui désiraient apprendre le français. En 2000, Normand Fortin, un coopérant qui avait étudié au Collège, avait organisé un programme d'été d'immersion sous la houlette de l'Université de l'Alberta (Faculté Saint-Jean et Centre d'enseignement et de recherche en français) à l'intention des étudiants canadiens (Kuntz et Michaels, 2001).

L'accès à des bibliothèques est très important pour les étudiants en langue. À La Pocatière, ces derniers pouvaient utiliser des ressources documentaires importantes pour leurs recherches ou, simplement, pour leurs lectures.

Le présent texte constitue un résumé des entrevues auxquelles se sont prêtés les directeurs des différentes bibliothèques de la ville. Formulées dans le but d'améliorer le programme d'immersion et faire en sorte que des liens ou des échanges s'établissent entre ces directeurs, les étudiants et les professeurs, les questions portaient sur les locaux, les employés, les collections et les services.

\section{Revue de la documentation}

Avant d'entreprendre les entrevues avec les directeurs des bibliothèques, j'ai effectué une revue de la documentation existante sur l'histoire des bibliothèques en sol québécois et à La Pocatière, de même que sur les programmes d'immersion. 


\section{Quand la Fondation Carnegie (des États-Unis) fit une offre de financer la construction de bibliothèques, les élites et les prêtres du Québec la déclinèrent par crainte du militantisme protestant et de l'influence de la langue anglaise.}

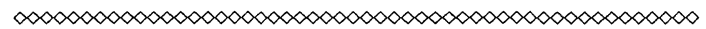

\section{Histoire des bibliothèques québécoises}

Les bibliothèques québécoises ne se sont pas développées aussi rapidement qu'au Canada pour plusieurs raisons. Il y existait bien des bibliothèques, mais elles n'étaient pas toujours ouvertes au lectorat extérieur. L'habitude de la lecture n'était pas non plus chose courante dans les foyers. Les prêtres achetaient des livres, mais on peut dire qu' ils les mettaient à disposition d'une manière très prudente (Baillargeon, 2005; Baillargeon, 1992; Brault, 2004; Chartrand, 1977; Kuntz, 2001; Lajeunesse, 2005; McNally, 2002; Savard, 1997). En plus d'en rester les uniques gardiens, ils ne se procuraient, la plupart du temps, que les livres recommandés par les autorités catholiques et ils évitaient soigneusement ceux qui étaient mis à l'Index, identifiés dans l'Index Librorum Prohibitorum, publié depuis 1557. Quand la Fondation Carnegie (des États-Unis) fit une offre de financer la construction de bibliothèques, les élites et les prêtres du Québec la déclinèrent par crainte du militantisme protestant et de l'influence de la langue anglaise. Il faut préciser toutefois qu'il existait bien des bibliothèques publiques ou universitaires dans les grandes villes telles que Trois-Rivières, Québec ou Montréal, mais la plupart appartenaient à des communautés religieuses ou relevaient d'institutions d'enseignement ou autres. En raison de l'apparition tardive, après 1960, des bibliothèques publiques dans les plus petites villes ou les villages, on ne trouve que peu d'articles historiques (Brault, 2004). Par contre, Bergeron, Deschatelets et Nauche (1998) ont décrit la situation, le statut, les habitudes et les comportements des employés de bibliothèques de lecture publique. De manière générale, ils ont relevé le fait que les bibliothécaires des communautés rurales participaient peu aux activités ou aux réseaux professionnels.

\section{Histoire des bibliothèques à La Pocatière}

Il n'existe que peu de documents consacrés aux bibliothèques pocatoises. L'histoire du Collège de SainteAnne est cependant plus étoffée. Lebon (1948b) a décrit comment, en 1887, l'abbé Charles Trudelle a transféré les documents gardés dans un petit local du deuxième étage dans une bibliothèque plus grande située sous la coupole du collège. Ainsi peut-on lire :
«... l'abbé Dominique Pelletier fait remarquer que la bibliothèque générale qui compte déjà plus de 12,00o volumes, à part la bibliothèque des séminaristes de celle des élèves, s'est augmentée cette année-là de plusieurs ouvrages dûs surtout à la générosité de MM. les abbés Charles Bacon, Georges Beaulieu, Chs Galarneau et C. A. Collet. Il ajoute que le bibliothécaire s'occupe de refaire et de compléter le catalogue. Il adressera à plusieurs amis des études et des livres, une liste des doubles à vendre ou à échanger."

Lebon, $1948 \mathrm{~b}: 82$

Quant à François Gagnon (1976), il a dépeint les activités du bibliothécaire Charles Bourque pendant la première partie $\mathrm{du} \mathrm{xx}^{\mathrm{e}}$ siècle, lequel avait exercé son métier avec grand enthousiasme.

«M. Charles Bourque, pendant 20 ans, s'y révéla un bibliothécaire compétent pour l'époque, connaissait bien le département où il évoluait et toujours prêt à faire profiter ses confrères de son expérience. Courtois, d'une distinction qui ne se démentit jamais, il recevait chacun avec plaisir, et tous paraissaient satisfaits de ses services. Bien peu se doutaient de la vie douloureuse que dissimulait cette amabilité. Il tint le coup jusqu'en 1951. »

$$
\text { Gagnon, } 1976 \text { : 130-31 }
$$

En 1967 , le bibliothécaire du Collège avait organisé une conférence sur les problèmes liés à la traduction de la Classification décimale de Dewey (Guillien, 1977). Aux Archives de la Côte-du-Sud et au Collège de Sainte-Anne, on trouve des photos de la bibliothèque du Collège, du Cégep de La Pocatière et de l'Institut de technologie agroalimentaire (ITA) que l'on peut aller voir en ligne, de même que trois photos des anciennes bibliothèques tirées d'un cahier de cartes postales que possède le Collège. Malheureusement, avec la mort de chaque prêtre a disparu une parcelle de la mémoire et de l'histoire de l'établissement. Parmi les bibliothécaires qui s'y sont succédé, on compte Raymond Boucher, Réal-Léonce Lévesque, Simon Tremblay et Marcel Mignault (depuis 1975). Avant l'arrivée de JeanLouis Demers, Thérèse Lambert (1974-1983) avait dirigé le cégep, et Marie Goyette la remplaça (1984-1989).

Pour ce qui est de l'ITA, Paul Venne (1985), dans un article sur l'état de l'agriculture québécoise, y parle de la bibliothèque. Dans les années 1950, on avait entrepris d'organiser la bibliothèque à l'aide d'un schéma de classification. Un bibliothécaire itinérant fut recruté qui visitera les stations agricoles et celle de La Pocatière, et y maintiendra la collection. Plus tard, c'est dans le laboratoire de la ferme expérimentale de La Pocatière, qui a ouvert ses portes en septembre 1985, qu'on installa une bibliothèque moderne. On y avait prévu suffisamment de rayonnages pour accueillir les collections et les abonnements supplémentaires résultant de l'accroissement 
du personnel de recherche, et on y a installé des ordinateurs.

Enfin, en ce qui a trait à la Bibliothèque municipale, où le «président » change presque chaque année, seules ses activités font l'objet d'un rapport annuel à l'intention du conseil et du maire. Et, jusqu'à maintenant, personne n'en a écrit l'histoire.

\section{Méthodologie}

La réalisation de la présente enquête présentait un certain nombre de difficultés méthodologiques. Voici un aperçu des étapes suivies.

\section{Échantillon}

D’abord, cinq bibliothèques - ou établissements documentaires - ont été identifiées à l'intérieur. des frontières de la ville : celle du Collège de Sainte-Anne, la Bibliothèque François-Hertel (Cégep), celle de l'ITA, la Bibliothèque municipale et les Archives de la Côte-duSud. Il existait bien d'autres écoles et d'autres bibliothèques, mais étant fermées pendant les vacances d'été, on ne pouvait en interviewer les responsables. Ainsi a-t-il été impossible de visiter les bibliothèques de l'École élémentaire Sacré-Cour, de l'École Saint-Charles (Centre de formation professionnelle aux adultes), de l'École polyvalente (de niveau secondaire) ou encore celles des prématernelles et des garderies, comme l'École Montessori.

\section{Collecte des données}

La collecte des données s'est faite en trois étapes. Avant de réaliser les entrevues, je me suis familiarisée avec le vocabulaire propre à la bibliothéconomie (RollandThomas, 1969). Ensuite, au cours de la première semaine de programme, j'ai procédé à l'interview (en personne) de chaque directeur des cinq établissements. Le questionnaire informel comportait les sujets suivants :

- L'année de fondation de la bibliothèque ;

- Le nombre d'employés et le type d'emploi ;

- Le système de classification des documents ;

- La personne chargée des prêts de livres et du prêt entre bibliothèques ;

- Le nombre d'ordinateurs et autres appareils ;

- La connectivité sans fil ;

- Le type de formation bibliographique ou bibliothéconomique;

- Les modes d'acquisition des documents;

- La périodicité de l'élagage ;

- La méthode de catalogage ;

- Les publications des directeurs ou des autres employés ;

- Les activités professionnelles du personnel ;

- La formation du directeur et de ses collaborateurs.

\section{Il existe une mémoire collective de l'établissement, car la bibliothèque profite des prestations de 73 bénévoles, lesquels occupent des " postes » par rotation.}

$\infty 0000000000000000000000000000000000$

À la fin des entrevues, les directeurs m'ont fait visiter les lieux, et on m'a autorisée à prendre des photos. La durée de chaque visite a été d'une heure ou plus. Dans certains cas, on m'a offert des dépliants publicitaires et donné des cartes professionnelles.

\section{Résultats}

Le profil des directeurs des bibliothèques observées à La Pocatière allait à l'encontre des stéréotypes. Quatre des cinq bibliothèques étaient dirigées par des hommes âgés de 40 à 60 ans, travaillant à temps plein et occupant le même poste depuis au moins dix ans. Tous avaient obtenu un baccalauréat, mais pas nécessairement en bibliothéconomie. La plupart d'entre eux avaient publié au moins un article ou un document.

La situation à la Bibliothèque municipale était différente. À ce moment-là occupée par une femme, la " présidence " y est assumée bénévolement - comme les autres employés également bénévoles - pour un mandat de deux ans, lequel n'est pas renouvelable. La " directrice " relève du maire et, à ce titre, elle doit lui présenter un rapport annuel. De plus, elle adressait un rapport trimestriel au journal local qui le publiait. Il existe une mémoire collective de l'établissement, car la bibliothèque profite des prestations de 73 bénévoles, lesquels occupent des "postes » par rotation. Tantôt, celui qui s'occupait de publicité hérite de l'élagage et celui qui faisait les acquisitions se voit confier la responsabilité des expositions, etc.

\section{Employés}

Toutes les bibliothèques du territoire avaient plus d'un employé. Celle du cégep avait le plus grand nombre d'employés permanents, soit six. En revanche, la Bibliothèque municipale comptait de nombreux bénévoles assumant les différentes fonctions comme la référence, les acquisitions, le catalogage ou le service de prêt. Dans les bibliothèques des écoles post-secondaires, les employés travaillent à temps plein durant l'année scolaire. Dès lors, les étudiants du programme d'immersion peuvent avoir beaucoup d'aide. Malheureusement, le directeur de la bibliothèque du Collège et les employés du cégep prennent leurs vacances pendant le déroulement du programme. 


\section{En raison des différences entre les trois systèmes, les étudiants $d u$ programme d'immersion ont besoin d'une séance d'information afin de leur faciliter le repérage les documents.}

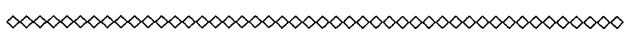

\section{Classification}

La plupart des bibliothèques utilisent le système de classification décimale Dewey. Cependant, au Canada, les œuvres littéraires sont cataloguées avec un [C] et on les place sur les rayons après les autres livres de la même cote. Par exemple, le roman Aurore d'André Mathieu porte la cote [C848. $\left.\mathrm{M}_{431} \mathrm{Au}\right]$ et non [848.M431 Au]. Le deuxième chiffre est l'indice Cutter : $M$ pour Mathieu suivi du chiffre d'auteur donné par la table de Cutter.

Par contre, les documents de l'ITA sont classés d'après le système Library of Congress. Par exemple, le roman Aurore est catalogué [PS8576A826], [PS] désignant la littérature canadienne. Le chiffre [8576] est assigné à André Mathieu. Les lettres et les chiffres qui suivent sont pour le titre Aurore, c'est-à-dire A pour Aurore et l'indice correspondant aux lettres qui suivent.

Aux Archives de la Côte-du-Sud, il y a un système de classement établi selon les patronymes de la région. On y trouve des noms comme Roland Martin, Raymond Boutet, Arthur Gendreau, Maurice Proulx et la famille Destroismaisons. Les documents ne sont pas tous inventoriés. Les rayons sont fermés au public.

En raison des différences entre les trois systèmes, les étudiants du programme d'immersion ont besoin d'une séance d'information afin de leur faciliter le repérage les documents.

\section{Le prêt}

Le prêt est un contrat tacite par lequel une bibliothèque permet à un lecteur ou emprunteur l'usage d'un livre, d'une livraison de périodique ou autre document durant une période déterminée, sans frais, mais à charge de restitution. Le nombre de livres que l'on peut emporter chez soi de même que la durée de l'emprunt varient d'un établissement à l'autre.

Au cours de l'été, les horaires diffèrent de l'horaire habituel. Pendant que certains employés sont en vacances, d'autres ont des horaires de travail réduits. Quant aux règlements du prêt, ils varient d'un endroit à l'autre. Ainsi, à la bibliothèque du cégep, le prêt est ouvert seulement aux étudiants qui y sont inscrits. Les personnes de l'extérieur, et c'est le cas des étudiants du programme d'immersion, peuvent cependant consulter et utiliser les documents sur place.

Par contre, à la bibliothèque de l'ITA et à celle du Collège Sainte-Anne, le prêt est ouvert à tous. Malheu- reusement, peu de gens à La Pocatière le savent. On peut $\mathrm{y}$ emprunter des documents de deux à quatre semaines. C'est dire que les étudiants peuvent recourir au prêt à ces deux bibliothèques pendant le cours d'immersion.

À la Bibliothèque municipale, seuls les habitants de la ville peuvent obtenir une carte d'abonné. Ainsi, il est interdit d'emprunter des livres aux étudiants ou aux élèves qui n'y résident pas, mais le travail et la consultation sur place leur est permis.

Enfin, les Archives sont ouvertes aux chercheurs. Mais on doit consulter les documents sur les lieux. Bref, même si les établissements documentaires ont des politiques ou des pratiques différentes en ce qui a trait au prêt, les étudiants peuvent néanmoins profiter des autres services qui y sont offerts.

\section{Types de catalogues}

La plupart des bibliothèques de la ville ont un catalogue en ligne. C'est le cas des bibliothèques municipale, du cégep et de l'ITA, ainsi que des Archives. Toutefois, elles recourent à des systèmes différents : l'ITA et le cégep utilisent « Regard » et la Bibliothèque municipale, "Taos». Les commandes sont un peu différentes d'un système à l'autre.

Le Collège, lequel possède la collection la plus importante, a toujours un catalogue sur fiches. On y trouve les trois types traditionnels de fiches : auteur, titre et sujet. Bien que les usagers plus âgés savent utiliser ce système, les plus jeunes ont de la difficulté à le comprendre. Puisque peu de bibliothèques d'université ont encore des fichiers, les étudiants se sentent frustrés. Il en va de même aux Archives.

\section{Nombre d'ordinateurs}

Chaque bibliothèque de La Pocatière possède des ordinateurs, mais leur nombre en varie d'un établissement à l'autre. La bibliothèque du cégep en a 13, ainsi que plusieurs scanners (numériseurs à balayage) et imprimantes. À l'ITA, il y a quatre ordinateurs pour les usagers et deux autres réservés aux employés. Au Collège, deux ordinateurs sont mis à la disposition du public, mais pas d'imprimante ni de scanner.

En comparaison, les deux ordinateurs mis à la disposition des lecteurs, à la Bibliothèque municipale, servent uniquement de catalogue. Cependant, les abonnés peuvent avoir accès à Internet durant quelques minutes seulement, ne disposant que de peu de temps pour lire leur courrier électronique. Aussi, un autre ordinateur, branché directement aux Archives, permet de visualiser les photos. Les bénévoles disposent de quatre ordinateurs pour la gestion, c'est-à-dire la référence, le prêt entre bibliothèques, les prêts et pour d'autres tâches.

Aux Archives, seul le directeur a un ordinateur à sa disposition. Cependant, de temps en temps, les chercheurs peuvent utiliser l'appareil. 
En général donc, le nombre d'ordinateurs est restreint par rapport au nombre d'étudiants du programme d'immersion. En conséquence, peu d'étudiants utilisent les bibliothèques pour leur correspondance électronique ou faire leurs devoirs.

\section{Formation et information documentaire}

Tous les directeurs ou président ont affirmé avoir un programme de formation documentaire destiné à leur lectorat. Les responsables de l'ITA, du cégep ou du Collège offrent un programme permanent au cours de l'année scolaire, lequel est destiné aux étudiants et à leurs instructeurs ou professeurs. De plus, on met plusieurs guides à la disposition des étudiants (exemple : "Description bibliographique ").

$\mathrm{Au}$ Collège, le bibliothécaire Marcel Mignault a conçu plusieurs guides pour faciliter la recherche documentaire des élèves du secondaire et même du primaire. Et, de fait, les bibliothécaires scolaires continuent d'en demander des exemplaires ou des copies. Malgré cela, les étudiants ont manqué d'informations importantes, car les instructeurs du cours d'immersion n'utilisent pas ces documents puisqu'ils en ignorent l'existence.

À la Bibliothèque municipale, les bénévoles initient les élèves des écoles maternelles, élémentaires et secondaires. Au cours de l'été, on offre un programme de lecture pour les jeunes. Peut-être, pourrait-on offrir une présentation sur les livres d'images pour les élèves débutants.

L'information documentaire, aux Archives, est donnée sur place, au téléphone ou par courrier électronique. Quelques étudiants ont visité les Archives et discuté avec le directeur. Il serait utile que les étudiants des cours avancés ou intermédiaires puissent visiter les bibliothèques pour s'y familiariser et enrichir leur vocabulaire dans le domaine.

\section{Collections spéciales}

Les bibliothèques de La Pocatière possèdent des collections spéciales.

$\mathrm{Au}$ Collège, il existe trois importantes collections spéciales : les livres anciens en latin et en grec, les périodiques anciens et les manuels scolaires du niveau secondaire. Les prêtres y ont donné leurs collections personnelles au cours des années. Bien que les étudiants en immersion n'aient pas besoin de consulter ces livres, il est intéressant d'en connaître l'existence. Par ailleurs, on y trouve également une collection de livres d'images, laquelle pourrait être utile aux élèves débutants.

La collection spéciale de l'ITA est composée de documents scientifiques. Comme la plus grande partie de la collection est en anglais, elle ne répond pas vraiment aux besoins des étudiants des classes d'immersion française.

Du côté du cégep, on trouve une intéressante collection de bandes dessinées qui pourrait être utile

\section{Au Collège, le bibliothécaire Marcel Mignault a conçu plusieurs guides pour faciliter la recherche documentaire des élèves $d u$ secondaire et même du primaire.}

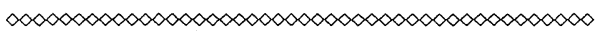

aux étudiants des groupes d'immersion. De plus, le cégep a de bonnes collections de vidéos ou de DVD. Les étudiants peuvent les visionner sur place, dans un local destiné à cet usage.

Aux Archives, les étudiants inscrits au programme d'immersion peuvent retracer l'histoire de familles implantées dans la région ou de leur propre famille. Il est très intéressant de s'attarder aux photos anciennes du Collège de Sainte-Anne.

Il y a une collection permanente à la Bibliothèque municipale. Composée de livres achetés grâce à une subvention de la Caisse populaire, elle appartient à la Ville.

\section{Élagage}

Élaguer une collection signifie en retirer les exemplaires en trop, les ouvrages superflus, rarement consultés ou vieillis, et placer ceux-ci dans une réserve ou un dépôt. Cette tâche relève essentiellement des bibliothèques. Aussi n'y a-t-il pas d'élagage aux Archives. À la Bibliothèque municipale, on procède à l'élagage périodiquement par le Réseau Biblio du Bas-Saint-Laurent, dans la mesure où les collections sont déplacées d'un établissement à l'autre. Les directeurs des bibliothèques s'acquittent de cette tâche généralement quand ils ont du temps libre. Les étudiants ignorent habituellement à quel moment cette opération est effectuée, à moins qu'ils ne fassent partie des bénévoles.

\section{Acquisitions}

Lacquisition désigne le processus suivi pour se procurer des livres et d'autres documents au moyen d'achats, d'échanges ou de dons. En général, les bibliothécaire font leur sélection en s'inspirant d'une politique d'acquisition modulée selon les besoins, par secteurs ou domaines. Pour ce qui est des périodiques, il s'agit de décider de renouveler ou d'annuler des abonnements. Il faut être prudent en la matière, bien vérifier les titres, car les magazines, revues et journaux coûtent cher. Généralement, les bibliothécaires utilisent les catalogues de périodiques pour faire leur choix. Les directeurs préparent les commandes de nouveaux livres sur des fiches préparées à cet effet. Dans la plupart des cas, cette tâche est réalisée avant la rentrée scolaire ou l'arrivée des étudiants. Le responsable du programme d'immersion peut recommander l'achat d'un certain nombre de 


\section{Les bibliothèques ont pu être considérées comme un élément essentiel du programme d'immersion.}

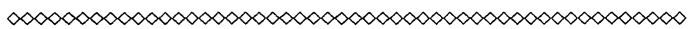

volumes, mais il est plus simple d'apporter les siens et de les mettre à disposition.

\section{Catalogage}

Comme c'est le cas pour les acquisitions, on procède au catalogage dans un local réservé à cet usage et les horaires de ce service peuvent y être différents des heures d'ouverture de l'établissement. Peu de bibliothécaires font eux-mêmes le catalogage des documents, un travail que l'on confie généralement à des techniciens ou techniciennes en documentation. Ceux-ci exécutent l'ensemble des opérations qui amènent tout livre ou tout document à l'étape où l'on peut l'utiliser sur place ou l'emprunter. Cela va de la pose de la puce de sécurité à la préparation de la fiche d'emprunt (à moins de disposer d'un système de prêt automatisé ou d'un catalogue en ligne) et à l'inscription de la cote.

Les bibliothécaires du cégep ou du Collège achètent les données catalographiques de Services documentaires multimedia. À l'ITA, on les obtient de la Bibliothèque du Congrès (Washington) ou de la Bibliothèque nationale du Canada. À la Bibliothèque municipale, on utilise le travail fait par les services du Réseau Biblio. La plupart des personnes responsables de ce service étaient en vacances au moment du programme d'immersion.

\section{Activités professionnelles}

Seulement deux bibliothécaires en poste avaient participé à des activités professionnelles. Les deux sont également membres d'une association (Demers, 2001; Mignault, 1976, 1979; Taillon). Dans le passé, ils ont écrit des articles ou des livres. L'un, Marcel Mignault, a organisé des réunions à l'intention des bibliothécaires scolaires au cours de l'année 1970. De son côté, la présidente de la Bibliothèque municipale (Doucet, 2005) écrit à tous les mois un article destiné au journal La Pocatoise. Les autres bibliothécaires aimeraient bien participer à une association, mais ils trouvent que cela coûte cher et prend beaucoup de temps. À noter que dans la plupart des cas, personne ne peut prendre le relais lorsqu'ils s'absentent.

\section{Conclusion}

Bien que toutes les bibliothèques diffèrent par leur gestion, leur prestation de services et leur équipement, les étudiants des cours d'immersion peuvent obtenir beaucoup de services et des informations dans ces cinq bibliothèques de La Pocatière. Les bibliothécaires sont très au fait de leurs collections et de leurs domaines de prédilection.

En dépit de ces ressources, il reste des zones d'amélioration possible, comme dans toutes les bibliothèques du Québec, d'ailleurs (Farhat et Girard, 2004). D'abord, il n'y a pas vraiment de collaboration établie entre les directeurs. Chacun sert d'abord son propre lectorat ou ses abonnés. Ensuite, le système de classification est différent d'un établissement à l'autre, il n'y a pas de prêt interbibliothèques et les catalogues n'offrent pas de liens électroniques avec les autres bibliothèques. Enfin, les heures d'ouverture sont limitées durant l'été à cause des vacances.

Aussi, la majorité des directeurs ne sont pas bibliothécaires et la maîtrise en bibliothéconomie y est l'exception. Souvent, ils ont eu une autre carrière, tantôt en histoire, en littérature ou en pédagogie. Quelques-uns sont néanmoins retournés à l'Université de Montréal afin d'obtenir un diplôme en bibliothéconomie et en sciences de l'information.

Le problème le plus sérieux est l'insuffisance des crédits budgétaires pour l'embauche d'employés, et pour l'achat de documents et d'équipement. Le budget de toutes les bibliothèques est habituellement modeste. Et les périodiques, souvent hors de prix. L'ITA, cependant, dispose de montants de fonctionnement suffisants, mais les autres bibliothèques luttent pour leur survie. Ce contexte affecte le programme d'immersion dans la mesure où l'on ne dispose pas de beaucoup de journaux, de documents visuels (vidéo ou DVD), de guides ou de fascicules destinés à faciliter l'utilisation des bibliothèques.

Néanmoins, les bibliothèques ont pu être considérées comme un élément essentiel du programme d'immersion. Par exemple, les étudiants ont participé au programme de lecture et ils ont lu des contes recommandés à la Bibliothèque municipale. Pour l'avenir, il faudrait que les directeurs des cinq bibliothèques de La Pocatière collaborent avec le personnel du programme d'immersion. (-)

\section{Sources consultées}

Baillargeon, Jean-Paul. 2005. « Les bibliothèques publiques et la Révolution tranquille au Québec ", Bulletin des bibliothèques de France, vol. 50, $\mathrm{n}^{\mathrm{O}} 1: 5-12$.

1992. "Les bibliothèques publiques du Québec, 1961-1989 ", Documentation et bibliothèques, vol. 38 , juilletseptembre : 139-147.

Bergeron, Pierrette, Gilles Deschalelets et Benédicte Nauche. 1998. "Les employés des bibliothèques francophones au Canada et leurs habitudes et pratiques de formation continue ", Documentation et bibliothèques, vol. $44, \mathrm{n}^{\circ} 1$, janvier-mars : 16-29.

Boucher, Raymond et Mignault, Marcel. 1978. Les étapes de la rédaction d'un travail en bibliothèque, La Pocatière, Société du stage en bibliothéconomie.

Boucher, Denis, Raymond Boucher et Marcel Mignault. 1969. Liste annotée d'ouvrages de consultation, ze éd. revue et augmentée, La Pocatière, Collège de Sainte-Anne-de-la-Pocatière. 5 vol. (Guides du personnel; 8.) 
Les bibliothèques à La Pocatière

\begin{tabular}{|c|c|c|c|c|c|}
\hline & $\begin{array}{l}\text { Archives de la Côte- } \\
\text { du-Sud (Société } \\
\text { hist.) }\end{array}$ & $\begin{array}{l}\text { Institut technologie } \\
\text { agroalimentaire }\end{array}$ & $\begin{array}{l}\text { Cégep de La } \\
\text { Pocatière }\end{array}$ & $\begin{array}{l}\text { Collège de Sainte- } \\
\text { Anne }\end{array}$ & $\begin{array}{l}\text { Bibliothèque } \\
\text { municipale }\end{array}$ \\
\hline Employés/bénévoles & 3 & 3 & 6 & 1 & 73 bénévoles \\
\hline Type d'emploi & $\begin{array}{l}1 \text { temps plein } \\
2 \text { temps partiel }\end{array}$ & 3 temps plein & 6 temps plein & $\begin{array}{l}1 \text { temps plein } \\
1 \text { temps partiel }\end{array}$ & 73 temps partiel \\
\hline Horaire d'été & $\begin{array}{l}\text { Lundi-vendredi } \\
8 \mathrm{~h} 30-12 \mathrm{~h} \\
13 \mathrm{~h}-16 \mathrm{~h} 30\end{array}$ & $\begin{array}{l}\text { Lundi-vendredi } \\
7 \mathrm{~h}_{30}-17 \mathrm{~h}\end{array}$ & $\begin{array}{l}\text { Lundi-vendredi } \\
8 \mathrm{~h} 30-12 \mathrm{~h} \\
13 \mathrm{~h} 30-16 \mathrm{~h} \\
(\text { Vac. }: 2 \mathrm{sem} .)\end{array}$ & $\begin{array}{l}\text { Lundi-vendredi } \\
9 \mathrm{~h}-12 \mathrm{~h} \\
13 \mathrm{~h}-16 \mathrm{~h} 30 \\
\text { (Vac. : } 2 \mathrm{sem} .)\end{array}$ & $\begin{array}{l}\text { Lundi-jeudi } \\
7 \mathrm{~h}-21 \mathrm{~h} \\
\text { Samedi } \\
15 \mathrm{~h}-17 \mathrm{~h}\end{array}$ \\
\hline Dépôt de livres & Non & Oui & Oui & Non & Non \\
\hline Classification & Maison & Library of Congress & Dewey & Dewey & Dewey \\
\hline Circulation & Pas de prêt & $\begin{array}{l}\text { Prêt aux étudiants et } \\
\text { aux citoyens }\end{array}$ & $\begin{array}{l}\text { Prêt aux étudiants et } \\
\text { aux professeurs }\end{array}$ & $\begin{array}{l}\text { Prêt aux étudiants, } \\
\text { profs. et citoyens }\end{array}$ & Prêt aux abonnés \\
\hline Prêt entre bibl. & Non & Oui & Oui & Non & Oui \\
\hline Porte électronique & Non & Oui & Oui & Non & Non \\
\hline Catalogue & Fiches et Web & Regard (en ligne) & Regard (en ligne) & Fiches & Taos (en ligne) \\
\hline Ordinateurs (publ.) & 1 & 4 & 13 & 2 & 3 \\
\hline Connectivité sans fil & Non & Non & Oui & Non & Non \\
\hline Instruction & Chercheurs & Étudiants & Étudiants & Élèves & Programmes d'été \\
\hline Acquisitions & Dons & Achat & Achat & Achat & Réseau Biblio -RdL \\
\hline Élagage & Non & À l'occasion & Un peu & Non & Tous les trois mois \\
\hline Catalogage & Directeur & Technicienne & Technicienne & Technicienne & Réseau Biblio - RdL \\
\hline Publications & Oui & & Oui & Oui & Non \\
\hline Activités prof. & Non & Non & Oui & Oui & Non \\
\hline Date & 1990 & $1990(1859)$ & 1964 & $1881-1962(1827-1841)$ & 1987 \\
\hline Années de travail & 15 & 15 & 10 & 10 & \\
\hline $\begin{array}{l}\text { Diplôme(s) des } \\
\text { directeurs }\end{array}$ & BA histoire & BA français & $\begin{array}{l}\text { BA littérature } \\
\text { Certificat en } \\
\text { biblothéconomie }\end{array}$ & $\begin{array}{l}\text { BA-littérature } \\
\text { BS-pédagogie } \\
\text { Certificat en } \\
\text { bibliothéconomie }\end{array}$ & \\
\hline
\end{tabular}

Brault, Jean-Rémi. 2004. "Regard sur l'évolution des bibliothèques québécoises : récit d'un professionnel ", Documentation et bibliothèques, vol. 50, $\mathrm{n}^{\circ} 3$, juillet-septembre : 213-232.

Chartrand, Georges A. 1977. Livres, bibliothèques et culture québécoise, Montréal, ASTED. 2 vol.

Demers, Jean-Louis. 2001. "L'interface humaine dans les services documentaires, une présence fragile à réinventer ", Pédagogie collégiale, vol. $15, \mathrm{n}^{\circ} 2: 24$.

Doucet, Maire-France. 2005. "Cet été, explorez l'espace de la lecture! ", La Pocatoise, vol. 9, n"12, 16 juillet.

Farhat, Jihad et Luc Girard. 2004. "L'avenir des services de référence des bibliothèques universitaires ", Argus, Montréal, vol. 33, n" 2, août :23-28.

Gagnon, François. 1976. "L'Alma mater ", La Pocatière, Collège de Sainte-Anne.

Guillien, Geneviève. 1977. « Le problème de la traduction dans ses rapports avec l'édition française de la Classification Décimale de Dewey ", [The problem of translation in relation to the French edition of the Dewey Decimal Classification], Dewey International : European Centenary Seminar on the Dewey Decimal Classification, Banbury, Angleterre, 26-30 septembre 1976, Joel C. Downing et M. Yelland, dir., Londres, Library Association, Forest Press and the Library Association, p. 60-66.

Kuntz, Patricia S. 2001. "A political history of public libraries in Quebec " (Bibliothèques municipales au Québec), ED 456860.

Kuntz, Patricia S. et Judith M. Michaels. 2001. "In search of professional development : North American Immersion Programs for teachers of French ", ED 459626.
Lajeunesse, Marcel. 2005. «Le bibliothécaire québécois : d'un homme de lettres à un professionnel de l'information ", Documentation et bibliothèques, vol. 48, $\mathrm{n}^{\prime \prime} 1: 125-135$.

Lebon, Wilfred. 1948. Histoire du Collège de Sainte-Anne-de-la-Pocatière. t. I : Le premier demi-siècle, T. 2 : Le second demi-siècle, Québec, Charrier \& Dugal.

McNally, Peter F. 2002. "Libraries in Canada : A précis ", Feliciter, vol. $48, \mathrm{n}^{\circ} 2: 75-77$.

Mignault, Marcel. 1979. Les chemins du savoir : La bibliothèque, la documentation, les habitudes de travail, t. I : Classes de secondaire 1 et 2; t. II : Classes de secondaire 3 et 5 , La Pocatière, Société du stage en bibliothéconomie.

Mignault, Marcel. 1976. Lise et Bruno dans l'univers des livres, La Pocatière, Société du stage en bibliothéconomie.

Rolland-Thomas, Paule, Victor Coulombe et Juliette Chabot. 1969. Vocabulaire technique de la bibliothéconomie et de la bibliographie, Montréal, Association canadienne des bibliothécaires de langue française.

Savard, R. 1997. "Le discours sur la lecture et l'évolution des bibliothèques publiques au Québec de 1850 à 1950 ", Argus, vol. 26, $\mathrm{n}^{\mathrm{N}} 2$, août : 19-27.

Séguin, Claire. 2000. "La littérature d'enfance et de jeunesse en mémoire ", À rayons ouverts, vol. 13, n" 51, juillet-septemvre : 4-5.

Venne, Paul R. 1985. "Les services de bibliothèque d'Agriculture Canada au Québec; bref historique ", Argus, vol. 14:131-134 